Article

\title{
Analysis of a Time-Fractional Substantial Diffusion Equation of Variable Order
}

\author{
Xiangcheng Zheng ${ }^{1}$, Hong Wang ${ }^{2}$ and Xu Guo ${ }^{3, *}$ \\ 1 School of Mathematical Sciences, Peking University, Beijing 100871, China; zhengxch@math.pku.edu.cn \\ 2 Department of Mathematics, University of South Carolina, Columbia, SC 29208, USA; hwang@math.sc.edu \\ 3 Geotechnical and Structural Engineering Research Center, Shandong University, Jinan 250100, China \\ * Correspondence: guoxu@sdu.edu.cn
}

check for updates

Citation: Zheng, X.; Wang, H.; Guo, $X$. Analysis of a Time-Fractional Substantial Diffusion Equation of Variable Order. Fractal Fract. 2022, 6, 114. https://doi.org/10.3390/ fractalfract6020114

Academic Editor: Haci Mehmet Baskonus

Received: 5 January 2022

Accepted: 10 February 2022

Published: 15 February 2022

Publisher's Note: MDPI stays neutral with regard to jurisdictional claims in published maps and institutional affiliations.

Copyright: (C) 2022 by the authors. Licensee MDPI, Basel, Switzerland. This article is an open access article distributed under the terms and conditions of the Creative Commons Attribution (CC BY) license (https:// creativecommons.org/licenses/by/ $4.0 /)$.
Abstract: A time-fractional substantial diffusion equation of variable order is investigated, in which the variable-order fractional substantial derivative accommodates the memory effects and the structure change of the surroundings of the physical processes with respect to time. The existence and uniqueness of the solutions to the proposed model are proved, based on which the weighted highorder regularity of the solutions, in which the weight function characterizes the singularity of the solutions, are analyzed.

Keywords: time-fractional diffusion equation; fractional substantial derivative; variable-order; existence and uniqueness; regularity

\section{Introduction}

Fractional problems with substantial derivatives have attracted increasing amounts of attention due to their applications in, e.g., characterizing the functionals of anomalous diffusion and non-Brownian motions [1,2]. There are several investigations in mathematical analysis and numerical methods to such kinds of problems [3-9], while the corresponding studies for the variable-order fractional substantial derivative models, in which the variable fractional order accommodates, e.g., the structure change of the surroundings with respect to time, are rarely found in the literature.

In some recent works, the following Caputo variable-order time-fractional diffusion equations are investigated mathematically and numerically [10-16]

$$
\left(\partial_{t}+q \partial_{t}^{\alpha(\cdot, t)}-\Delta\right) u(x, t)=f(x, t)
$$

Here, the Caputo variable-order fractional derivative operator is defined as [17-19]

$$
\partial_{t}^{\alpha(\cdot, t)} g(t):=\int_{0}^{t} \frac{(t-s)^{-\alpha(s, t)}}{\Gamma(1-\alpha(s, t))} \partial_{s} g(s) d s .
$$

The Caputo (variable-order) fractional derivative is widely used in various applications that exhibit the power law type memory effects due to the power function integral kernel. The Caputo variable-order fractional substantial derivative operator $\sigma \partial_{t}^{\alpha(\cdot, t)}$, which is defined in the following for some $\sigma \geq 0$ and $0 \leq \alpha(s, t)<1[1-3]$

$$
\sigma \partial_{t}^{\alpha(\cdot, t)} g(t):=\int_{0}^{t} \frac{(t-s)^{-\alpha(s, t)} e^{-\sigma(t-s)}}{\Gamma(1-\alpha(s, t))}\left(\partial_{s}+\sigma\right) g(s) d s,
$$

tempers the memory effects of the Caputo fractional derivative operator by introducing the exponential factor, such that the impacts located at $s$ are negligible if $t-s$ is large [1,3]. Due 
to the complicated form of the variable-order fractional substantial derivative, existing analysis techniques for investigating the Caputo variable-order fractional derivative problems may not apply directly for problems involving (1), which motivates the current work.

In this paper, we analyze the following time-fractional diffusion equation [20-28] equipped with variable-order fractional substantial derivative

$$
\begin{gathered}
\left(\partial_{t}+q^{\sigma} \partial_{t}^{\alpha(\cdot, t)}-\Delta\right) u(x, t)=f(x, t), \quad(x, t) \in \Omega \times(0, T] ; \\
u(x, 0)=u_{0}(x), x \in \Omega ; \quad u(x, t)=0,(x, t) \in \partial \Omega \times[0, T],
\end{gathered}
$$

where $q \geq 0, T>0, \Omega$ is a $d$-dimensional domain for $1 \leq d \leq 3$ with a smooth boundary $\partial \Omega$. In the following two sections, we rigorously prove the well-posedness and high-order regularity of the solutions to problem (2), respectively, which provide a theoretical basis for this model. In particular, a weight function is introduced in the estimate of high-order smoothing properties to characterize the initial singularity of the solutions.

In the rest of the paper, let $H^{1}(0, T)$ be the Hilbert space of functions with weak derivatives up to order 1 in $L^{2}(0, T)$, the space of square-integrable functions on $[0, T]$. The corresponding norms are defined by

$$
\begin{gathered}
\|g\|_{L^{2}(0, T)}:=\left(\int_{0}^{T} g^{2}(t) d t\right)^{1 / 2}, \\
\|g\|_{H^{1}(0, T)}:=\left(\|g\|_{L^{2}(0, T)}^{2}+\left\|\partial_{t} g\right\|_{L^{2}(0, T)}^{2}\right)^{1 / 2} .
\end{gathered}
$$

$H^{1}(0, T ; \mathcal{D})$ for some Banach space $\mathcal{D}$ stands for the space of the functions in $H^{1}(0, T)$ with respect to the norm of $\mathcal{D}$. $Q$ denotes a generic constant that may assume different values at different cases.

\section{Existence and Uniqueness}

We prove the existence and uniqueness of the solutions to problem (2) in this section. Throughout the paper, we assume that there exists a constant $0<\alpha^{*}<1$, such that

$$
0 \leq \alpha(s, t) \leq \alpha^{*} \text { for } 0 \leq s \leq t \leq T .
$$

Theorem 1. If $f \in L^{2}\left(0, T ; L^{2}(\Omega)\right)$ and $u_{0} \in \check{H}^{1}(\Omega)$, problem (2) has a unique solution $u \in$ $H^{1}\left(0, T ; L^{2}(\Omega)\right) \cap L^{2}\left(0, T ; \check{H}^{2}(\Omega)\right)$ and

$$
\|u\|_{H^{1}\left(0, T ; L^{2}(\Omega)\right)}+\|u\|_{L^{2}\left(0, T ; \breve{H}^{2}(\Omega)\right)} \leq Q\left(\|f\|_{L^{2}\left(0, T ; L^{2}(\Omega)\right)}+\left\|u_{0}\right\|_{\check{H}^{1}(\Omega)}\right)
$$

where $Q$ is independent from the data and the solutions and the norm $\|\cdot\|_{\check{H}^{p}(\Omega)}$ for $p \geq 0$ is defined in terms of the eigenpairs $\left\{\lambda_{i}, \phi_{i}(x)\right\}_{i=1}^{\infty}$ of $-\Delta$ by

$$
\|v\|_{\tilde{H}^{p}(\Omega)}^{2}:=\sum_{i=1}^{\infty} \lambda_{i}^{p}\left(v, \phi_{i}\right)^{2} .
$$

Proof. If we expand $u$ in terms of $\left\{\phi_{i}(\boldsymbol{x})\right\}_{i=1}^{\infty}$ as

$$
u(x, t)=\sum_{i=1}^{\infty} u_{i}(t) \phi_{i}(\boldsymbol{x}), u_{i}(t)=\left(u, \phi_{i}\right),
$$

then $\left\{u_{i}(t)\right\}_{i=1}^{\infty}$ satisfy the following ordinary differential equation

$$
\partial_{t} w(t)+q^{\sigma} \partial_{t}^{\alpha(\cdot, t)} w(t)+\lambda w(t)=h(t), \quad t \in(0, T] ; \quad w(0)=w_{0}
$$


where $w, \lambda, h$ and $w_{0}$ refer to $u_{i}, \lambda_{i},\left(f, \phi_{i}\right)$ and $\left(u_{0}, \phi_{i}\right)$, respectively, for $i \geq 1$. We consider the fractional term as part of the right-hand side term in (3) to obtain

$$
\partial_{t} w(t)+\lambda w(t)=-q^{\sigma} \partial_{t}^{\alpha(\cdot, t)} w(t)+h(t) .
$$

An application of the variation-of-constants formula yields

$$
w=e^{-\lambda t} w_{0}+e^{-\lambda t} *\left(-q^{\sigma} \partial_{t}^{\alpha(\cdot, t)} w+h\right)
$$

where $*$ stands for the convolution on $[0, t]$ as follows

$$
g_{1}(t) * g_{2}(t):=\int_{0}^{t} g_{1}(s) g_{2}(t-s) d s
$$

Then, we differentiate (4) with respect to $t$, multiply $e^{-\kappa t}$ for some $\kappa \geq 0$ on the resulting equation and reformulate $e^{-\kappa t} \cdot \sigma \partial_{t}^{\alpha(\cdot, t)}$ as a sum of a linear operator $\mathcal{S}$ and a function $\mathcal{H}(t)$

$$
\begin{aligned}
e^{-\kappa t} \cdot \sigma \partial_{t}^{\alpha(\cdot, t)} w:=\int_{0}^{t} \frac{(t-s)^{-\alpha(s, t)} e^{-(\sigma+\kappa)(t-s)}}{\Gamma(1-\alpha(s, t))} \\
\times\left(e^{-\kappa s} \partial_{s} w(s)+\sigma \int_{0}^{s} e^{-\kappa(s-y)} e^{-\kappa y} \partial_{y} w(y) d y+\sigma e^{-\kappa s} w_{0}\right) d s \\
\quad=: \mathcal{S}\left(e^{-\kappa t} \partial_{t} w\right)+\mathcal{H}(t), \\
\mathcal{H}(t):=\sigma w_{0} e^{-\kappa t} \int_{0}^{t} \frac{(t-s)^{-\alpha(s, t)} e^{-\sigma(t-s)}}{\Gamma(1-\alpha(s, t))} d s
\end{aligned}
$$

to obtain an integral equation in terms of $\tilde{w}:=e^{-\kappa t} \partial_{t} w$ as follows

$$
\begin{array}{r}
\tilde{w}=-\lambda e^{-(\lambda+\kappa) t} w_{0}+\left(-q \mathcal{S}(\tilde{w})-q \mathcal{H}(t)+e^{-\kappa t} h\right) \\
-\lambda e^{-(\lambda+\kappa) t} *\left(-q \mathcal{S}(\tilde{w})-q \mathcal{H}(t)+e^{-\kappa t} h\right) .
\end{array}
$$

In order to prove the well-posedness of this integral equation in $L^{2}(0, T)$, it suffices to show that the linear operator $-q S+\lambda q e^{-(\lambda+\kappa) t} * \mathcal{S}$ is a contraction in $L^{2}(0, T)$. For $v \in$ $L^{2}(0, T)$, we apply

$$
\left|(t-s)^{-\alpha(s, t)}\right|=(t-s)^{-\alpha^{*}}(t-s)^{\alpha^{*}-\alpha(s, t)} \leq \max \{1, T\}(t-s)^{-\alpha^{*}}
$$

and Young's convolution inequality to bound $\mathcal{S} v$ by

$$
\begin{aligned}
& \|\mathcal{S} v\|_{L^{2}(0, T)} \\
& \leq Q\left\|\int_{0}^{t} \frac{e^{-(\sigma+\kappa)(t-s)}}{(t-s)^{\alpha^{*}}}\left(|v(s)|+\sigma \int_{0}^{s} e^{-\kappa(s-y)}|v(y)| d y\right) d s\right\|_{L^{2}(0, T)} \\
& \leq\left. Q\left\|e^{-(\sigma+\kappa) t} t^{-\alpha^{*}}\right\|\right|_{L^{1}(0, T)}\|v\|_{L^{2}(0, T)} \\
& \leq Q(\sigma+\kappa)^{-\left(1-\alpha^{*}\right)}\|v\|_{L^{2}(0, T)} .
\end{aligned}
$$

The operator $\lambda e^{-(\lambda+\kappa) t} * \mathcal{S}$ is then accordingly bounded by

$$
\left\|\lambda e^{-(\lambda+\kappa) t} * \mathcal{S} v\right\|_{L^{2}(0, T)} \leq\|\mathcal{S} v\|_{L^{2}(0, T)} \leq Q(\sigma+\kappa)^{-\left(1-\alpha^{*}\right)}\|v\|_{L^{2}(0, T)} .
$$


We incorporate the above two equations to conclude that for $\kappa$ large enough, $-q S+$ $\lambda q e^{-(\lambda+\kappa) t} * \mathcal{S}$ is a contraction in $L^{2}(0, T)$ and, thus, the integral Equation (5) has a unique solution $\tilde{w} \in L^{2}(0, T)$. We further apply this contractability on (5) to obtain a stability estimate

$$
\begin{aligned}
\|\tilde{w}\|_{L^{2}(0, T)} & \leq Q\left(\lambda^{1 / 2}\left|w_{0}\right|+\|\mathcal{H}\|_{L^{2}(0, T)}+\|h\|_{L^{2}(0, T)}\right) \\
& \leq Q\left(\lambda^{1 / 2}\left|w_{0}\right|+\|h\|_{L^{2}(0, T)}\right) .
\end{aligned}
$$

Then, the $w$ defined by

$$
w:=\int_{0}^{t} e^{\kappa s} \tilde{w}(s) d s+w_{0} \in H^{1}(0, T)
$$

with the estimate

$$
\|w\|_{H^{1}(0, T)} \leq Q\|\tilde{w}\|_{L^{2}(0, T)} \leq Q\left(\lambda^{1 / 2}\left|w_{0}\right|+\|h\|_{L^{2}(0, T)}\right)
$$

satisfies the differential Equation (3). The unique $H^{1}$ solution to (3) follows from that of (5).

Based on the above derivations, we finally estimate the solution $u$ to problem (2) by

$$
\begin{aligned}
& \|u\|_{H^{1}\left(0, T ; L^{2}(\Omega)\right)}^{2} \leq Q \sum_{i=1}^{\infty}\left\|u_{i}\right\|_{H^{1}(0, T)}^{2} \leq Q \sum_{i=1}^{\infty}\left(\lambda_{i}\left|\left(u_{0}, \phi_{i}\right)\right|^{2}\right. \\
& \left.+\left\|\left(f, \phi_{i}\right)\right\|_{L^{2}(0, T)}^{2}\right)=Q\left(\|f\|_{L^{2}\left(0, T ; L^{2}(\Omega)\right)}^{2}+\left\|u_{0}\right\|_{H^{1}(\Omega)}^{2}\right) .
\end{aligned}
$$

The estimate of the solutions in $\|\cdot\|_{L^{2}\left(0, T ; \check{H}^{2}(\Omega)\right)}$ follows from

$$
\begin{aligned}
\|u\|_{L^{2}\left(0, T ; \check{H}^{2}(\Omega)\right)} & =\|\Delta u\|_{L^{2}\left(0, T ; L^{2}(\Omega)\right)} \\
& =\left\|\partial_{t} u+q^{\sigma} \partial_{t}^{\alpha(\cdot, t)} u-f\right\|_{L^{2}\left(0, T ; L^{2}(\Omega)\right)} \\
& \leq Q\left(\|f\|_{L^{2}\left(0, T ; L^{2}(\Omega)\right)}+\left\|u_{0}\right\|_{\check{H}^{1}(\Omega)}\right) .
\end{aligned}
$$

The uniqueness of the solutions to problem (2) follows from that of the differential Equation (3).

\section{Weighted Regularity} theorem.

We prove the weighted regularity of the solutions to problem (2) in the following

Theorem 2. Suppose $\alpha$ has bounded first-order derivatives for each coordinate, $u_{0} \in \check{H}^{s+3}(\Omega)$, $f \in L^{2}\left(0, T ; \breve{H}^{s+2}(\Omega)\right)$ and $\partial_{t} f \in L^{2}\left(0, T ; \breve{H}^{s}(\Omega)\right)$ for some $s \geq 0$. Then, the following estimate holds

$$
\begin{aligned}
& \left\|t^{\delta} \partial_{t}^{2} u\right\|_{L^{2}\left(0, T ; \check{H}^{s}(\Omega)\right)} \\
& \quad \leq Q\left(\left\|u_{0}\right\|_{\check{H}^{s+3}(\Omega)}+\left\|\partial_{t} f\right\|_{L^{2}\left(0, T ; \check{H}^{s}(\Omega)\right)}+\|f\|_{L^{2}\left(0, T ; \check{H}^{s+2}(\Omega)\right)}\right)
\end{aligned}
$$

where

$$
\delta:=\max \{\alpha(0,0)-1 / 2+\theta, 0\} \text { with } 0<\theta \ll 1
$$

and $Q$ is independent from the data and the solutions.

Proof. We differentiate (4) twice in time to obtain

$$
\begin{aligned}
\partial_{t}^{2} w= & \lambda^{2} e^{-\lambda t} w_{0}+\partial_{t}\left(-q^{\sigma} \partial_{t}^{\alpha(\cdot, t)} w+h\right) \\
& -\lambda\left(-q^{\sigma} \partial_{t}^{\alpha(\cdot, t)} w+h\right)+\lambda^{2} e^{-\lambda t} *\left(-q^{\sigma} \partial_{t}^{\alpha(\cdot, t)} w+h\right) .
\end{aligned}
$$


The $\partial_{t}^{\sigma} \partial_{t}^{\alpha(\cdot, t)} w$ could be simplified as

$$
\begin{aligned}
\left|\partial_{t}^{\sigma} \partial_{t}^{\alpha(\cdot, t)} w(t)\right| & =\left|\partial_{t} \int_{0}^{t} \frac{s^{-\alpha(t-s, t)} e^{-\sigma s}}{\Gamma(1-\alpha(t-s, t))}\left(\partial_{y}+\sigma\right) w(y)\right|_{y=t-s} d s \mid \\
& =\mid \frac{t^{-\alpha(0, t)} e^{-\sigma t}}{\Gamma(1-\alpha(0, t))}\left(\partial_{t} w(0)+\sigma w(0)\right) \\
+ & \left.\int_{0}^{t} \partial_{t}\left(\frac{s^{-\alpha(t-s, t)} e^{-\sigma s}}{\Gamma(1-\alpha(t-s, t))}\right)\left(\partial_{y}+\sigma\right) w(y)\right|_{y=t-s} d s \\
+ & \left.\int_{0}^{t} \frac{s^{-\alpha(t-s, t)} e^{-\sigma s}}{\Gamma(1-\alpha(t-s, t))}\left(\partial_{y}^{2}+\sigma \partial_{y}\right) w(y)\right|_{y=t-s} d s \mid \\
& \leq Q t^{-\alpha(0, t)}\left(\left|\partial_{t} w(0)\right|+|w(0)|\right)+Q \int_{0}^{t} \frac{\left|\partial_{s}^{2} u(s)\right|}{(t-s)^{\alpha(s, t)}} d s .
\end{aligned}
$$

As

$$
t^{-\alpha(0, t)}=t^{-\alpha(0,0)} t^{\alpha(0,0)-\alpha(0, t)} \leq Q t^{-\alpha(0,0)},
$$

we multiply $t^{\delta}$ on both sides of (7) and apply

$$
(t-s)^{\alpha(s, t)} \geq Q(t-s)^{\alpha(t, t)}
$$

to find

$$
\begin{aligned}
&\left|t^{\delta} \partial_{t}{ }^{\sigma} \partial_{t}^{\alpha(\cdot, t)} w(t)\right| \leq Q t^{\delta-\alpha(0,0)}\left(\left|\partial_{t} w(0)\right|+|w(0)|\right) \\
&+Q \int_{0}^{t} \frac{s^{\delta}\left|\partial_{s}^{2} u(s)\right|}{(t-s)^{\alpha^{*}}} d s+Q \int_{0}^{t} \frac{(t-s)^{\delta}\left|\partial_{s}^{2} u(s)\right|}{(t-s)^{\alpha(t, t)}} d s .
\end{aligned}
$$

Since

$$
\begin{aligned}
\int_{0}^{t} & \frac{(t-s)^{\delta}\left|\partial_{s}^{2} u(s)\right|}{(t-s)^{\alpha(t, t)}} d s \\
\quad & \int_{0}^{t} \frac{(t-s)^{\delta-\alpha(t, t) / 2}}{s^{\delta}} \frac{s^{\delta}\left|\partial_{s}^{2} u(s)\right|}{(t-s)^{\alpha(t, t) / 2}} d s \\
& \leq\left(\int_{0}^{t} \frac{(t-s)^{2 \delta-\alpha(t, t)}}{s^{2 \delta}} d s\right)^{1 / 2}\left(\int_{0}^{t} \frac{s^{2 \delta}\left|\partial_{s}^{2} u(s)\right|^{2}}{(t-s)^{\alpha(t, t)}} d s\right)^{1 / 2} \\
& \leq Q\left(t^{1-\alpha(t, t)}\right)^{1 / 2}\left(\int_{0}^{t} \frac{s^{2 \delta}\left|\partial_{s}^{2} u(s)\right|^{2}}{(t-s)^{\alpha(t, t)}} d s\right)^{1 / 2} \\
& \leq Q\left(\int_{0}^{t} \frac{s^{2 \delta}\left|\partial_{s}^{2} u(s)\right|^{2}}{(t-s)^{\alpha^{*}}} d s\right)^{1 / 2},
\end{aligned}
$$

we apply $\left\|e^{-\kappa t} \cdot\right\|_{L^{2}(0, T)}$ for some $\kappa>0$ on (8) to find

$$
\begin{aligned}
\left\|e^{-\kappa t} t^{\delta} \partial_{t} \partial_{t}^{\alpha(\cdot, t)} w(t)\right\|_{L^{2}(0, T)} \leq Q\left(\left|\partial_{t} w(0)\right|+|w(0)|\right) \\
+Q\left\|t^{-\alpha^{*}} e^{-\kappa t}\right\|_{L^{1}(0, T)}\left\|e^{-\kappa t} t^{\delta} \partial_{t}^{2} u\right\|_{L^{2}(0, T)} \\
+Q\left(\int_{0}^{T} \int_{0}^{t} \frac{e^{-2 \kappa(t-s)}}{(t-s)^{\alpha^{*}}}\left(e^{-\kappa s} s^{\delta} \partial_{s}^{2} u(s)\right)^{2} d s d t\right)^{1 / 2} \\
\leq Q\left(\left|\partial_{t} w(0)\right|+|w(0)|\right)+Q \kappa^{-\left(1-\alpha^{*}\right) / 2}\left\|e^{-\kappa t} t^{\delta} \partial_{t}^{2} u\right\|_{L^{2}(0, T)} .
\end{aligned}
$$

We, therefore, multiply $t^{\delta}$ on both sides of (6), apply $\left\|e^{-\kappa t} \cdot\right\|_{L^{2}(0, T)}$ on the resulting equation, choosing $\kappa$ large enough and employ (9) and $(t \lambda)^{\delta} e^{-\lambda t / 2} \leq Q$, as well as similar (and simpler) proofs as above to obtain

$$
\left\|e^{-\kappa t} t^{\delta} \partial_{t}^{2} w\right\|_{L^{2}(0, T)} \leq Q\left(\lambda^{3 / 2}\left|w_{0}\right|+\left\|\partial_{t} h\right\|_{L^{2}(0, T)}+\lambda\|h\|_{L^{2}(0, T)}\right) .
$$


We employ this result to obtain the estimate of $\partial_{t}^{2} u$ as

$$
\begin{aligned}
& \left\|t^{\delta} \partial_{t}^{2} u\right\|_{L^{2}\left(0, T ; \check{H}^{s}(\Omega)\right)}^{2}=\sum_{i=1}^{\infty} \lambda_{i}^{s}\left\|t^{\delta} \partial_{t}^{2} u_{i}\right\|_{L^{2}(0, T)}^{2} \\
& \quad \leq Q \sum_{i=1}^{\infty}\left(\lambda_{i}^{s+3}\left|\left(u_{0}, \phi_{i}\right)\right|^{2}+\left\|\left(\partial_{t} f, \phi_{i}\right)\right\|_{L^{2}(0, T)}^{2}+\lambda_{i}^{2}\left\|\left(f, \phi_{i}\right)\right\|_{L^{2}(0, T)}^{2}\right) \\
& \quad=Q\left(\left\|u_{0}\right\|_{\check{H}^{s+3}(\Omega)}^{2}+\left\|\partial_{t} f\right\|_{L^{2}\left(0, T ; L^{2}(\Omega)\right)}^{2}+\|f\|_{L^{2}\left(0, T ; \check{H}^{2}(\Omega)\right)}^{2}\right),
\end{aligned}
$$

which completes the proof.

\section{Conclusions}

In this paper we rigorously prove the well-posedness and high-order regularity of the solutions to the time-fractional diffusion equation equipped with variable-order fractional substantial derivative. In particular, a weight function is introduced in the estimate of high-order smoothing properties to characterize the initial singularity of the solutions. Based on these theoretical estimates, we will carry out numerical analysis for the proposed model in the near future in order to provide supports for practical implementations.

Author Contributions: X.Z.: Conceptualization, Methodology, Validation, Writing-Original Draft Preparation, Funding acquisition; H.W.: Conceptualization, Writing-Review and Editing, Funding acquisition; X.G.: Conceptualization, Writing—Review and Editing, Funding acquisition. All authors have read and agreed to the published version of the manuscript.

Funding: This work was partially funded by the ARO MURI Grant W911NF-15-1-0562, by the National Science Foundation under Grant DMS2012291, by the National Natural Science Foundation of China under Grant 11901354, 11971272, by the Natural Science Foundation of Shandong Province for Excellent Youth Scholars ZR2020YQ02, by Taishan Scholars Program of Shandong Province of China tsqn201909044, by the China Postdoctoral Science Foundation under Grants 2021TQ0017 and 2021M700244, and by the International Postdoctoral Exchange Fellowship Program (TalentIntroduction Program) YJ20210019.

Institutional Review Board Statement: Not applicable.

Informed Consent Statement: Not applicable.

Data Availability Statement: Not applicable.

Acknowledgments: The authors would like to express their most sincere thanks to the referees for their very helpful comments and suggestions, which greatly improved the quality of this paper.

Conflicts of Interest: The authors declare no conflict of interest.

\section{References}

1. Chen, M.; Deng, W. Discretized fractional substantial calculus. ESAIM M2AN 2015, 49, 373-394.

2. Friedrich, R.; Jenko, F.; Baule, A.; Eule, S. Anomalous diffusion of inertial, weakly damped particles. Phys. Rev. Lett. 2006, 96, 230601. [CrossRef] [PubMed]

3. Chen, M.; Deng, W. High order algorithms for the fractional substantial diffusion equation with truncated Lévy flights. SIAM J. Sci. Comput. 2015, 37, A890-A917. [CrossRef]

4. Deng, W.; Chen, M.; Barkai, E. Numerical algorithms for the forward and backward fractional Feynman-Kac equations. J. Sci. Comput. 2015, 62, 718-746. [CrossRef]

5. Deng, W. Finite element method for the space and time fractional Fokker-Planck equation. SIAM J. Numer. Anal. 2009, 47, 204-226. [CrossRef]

6. Fahad, H.; Rehman, M. Generalized substantial fractional operators and well-posedness of Cauchy problem. Bull. Malays. Math. Sci. Soc. 2021, 44, 1501-1524. [CrossRef]

7. Huang, C.; Zhang, Z.; Song, Q. Spectral methods for substantial fractional differential equations. J. Sci. Comput. 2018, 74, 1554-1574. [CrossRef]

8. Ortigueira, M.; Bengochea, G.; Machado, J. Substantial, tempered, and shifted fractional derivatives: Three faces of a tetrahedron. Math. Meth. Appl. Sci. 2021, 44, 9191-9209. [CrossRef]

9. Zayernouri, M.; Ainsworth, M.; Karniadakis, G. Tempered fractional Sturm-Liouville EigenProblems. SIAM J. Sci. Comput. 2015, 37, A1777-A1800. [CrossRef] 
10. Fu, Z.; Reutskiy, S.; Sun, H.; Ma, J.; Khan, M. A robust kernel-based solver for variable-order time fractional PDEs under 2D/3D irregular domains. Appl. Math. Lett. 2019, 94, 105-111. [CrossRef]

11. Wang, H.; Zheng, X. Wellposedness and regularity of the variable-order time-fractional diffusion equations. J. Math. Anal. Appl. 2019, 475, 1778-1802. [CrossRef]

12. Zhang, Y.; Green, C.; Baeumer, B. Linking aquifer spatial properties and non-Fickian transport in mobile-immobile like alluvial settings. J. Hydrol. 2014, 512, 315-331. [CrossRef]

13. Zheng, X.; Wang, H. A hidden-memory variable-order fractional optimal control model: Analysis and approximation. SIAM J Control Optim. 2021, 59, 1851-1880. [CrossRef]

14. Zheng, X.; Wang, H. Optimal-order error estimates of finite element approximations to variable-order time-fractional diffusion equations without regularity assumptions of the true solutions. IMA J. Numer. Anal. 2021, 41, 1522-1545. [CrossRef]

15. Zheng, X.; Wang, H. An error estimate of a numerical approximation to a hidden-memory variable-order space-time fractional diffusion equation. SIAM J. Numer. Anal. 2020, 58, 2492-2514. [CrossRef]

16. Zheng, X.; Wang, H. Analysis and numerical approximation to time-fractional diffusion equation with a general time-dependent variable order. Nonlinear Dyn. 2021, 104, 4203-4219. [CrossRef]

17. Lorenzo, C.F.; Hartley, T.T. Variable order and distributed order fractional operators. Nonlinear Dynam. 2002, 29, 57-98. [CrossRef]

18. Samko, S.; Ross, B. Integration and differentiation to a variable fractional order. Integral Transform. Spec. Funct. 1993, 1, 277-300. [CrossRef]

19. Sun, H.; Chang, A.; Zhang, Y.; Chen, W. A review on variable-order fractional differential equations: Mathematical foundations, physical models, numerical methods and applications. Fract. Calc. Appl. Anal. 2019, 22, 27-59. [CrossRef]

20. Hendy, A. Numerical treatment for after-effected multi-term time-space fractional advection-diffusion equations. Engrg. Comput. 2021, 37, 2763-2773. [CrossRef]

21. Jiang, S.; Zhang, J.; Zhang, Q.; Zhang, Z. Fast evaluation of the Caputo fractional derivative and its applications to fractional diffusion equations. Commun. Comput. Phys. 2017, 21, 650-678. [CrossRef]

22. Kumar, S.; Kumar, A.; Samet, B.; Gómez-Aguilar, J.; Osman, M. A chaos study of tumor and effector cells in fractional tumorimmune model for cancer treatment. Chaos Solitons Fractals 2020, 141, 110321. [CrossRef]

23. Kumar, S.; Kumar, R.; Osman, M.; Samet, B. A wavelet based numerical scheme for fractional order SEIR epidemic of measles by using Genocchi polynomials. Numer. Methods Partial Differ. Equ. 2021, 37, 1250-1268. [CrossRef]

24. Li, B.; Ma, S. Exponential convolution quadrature for nonlinear subdiffusion equations with nonsmooth initial data. SIAM J. Numer. Anal. 2022. [CrossRef]

25. Luchko, Y. Initial-boundary-value problems for the one-dimensional time-fractional diffusion equation. Fract. Calc. Appl. Anal. 2012, 15, 141-160. [CrossRef]

26. Mohammadi, H.; Kumar, S.; Rezapour, S.; Etemad, S. A theoretical study of the Caputo-Fabrizio fractional modeling for hearing loss due to Mumps virus with optimal control. Chaos Solitons Fractals 2021, 144, 110668. [CrossRef]

27. Sakamoto, K.; Yamamoto, M. Initial value/boundary value problems for fractional diffusion-wave equations and applications to some inverse problems. J. Math. Anal. Appl. 2011, 382, 426-447. [CrossRef]

28. Veeresha, P.; Prakasha, D.; Kumar, S. A fractional model for propagation of classical optical solitons by using nonsingular derivative. Math. Meth. Appl. Sci. 2020. [CrossRef] 